\title{
Biochemical nutrient requirements of the rotifer Brachionus calyciflorus: co-limitation by sterols and amino acids
}

\author{
Alexander Wacker,1 and Dominik Martin-Creuzburg ${ }^{2}$ \\ ${ }^{1}$ Institute of Biochemistry and Biology, University of Potsdam, Am Neuen Palais 10, 14469, Potsdam, Germany; and \\ ${ }^{2}$ Limnological Institute, University of Konstanz, Mainaustrasse 252, 78464, Konstanz, Germany
}

\begin{abstract}
Summary
1. It has been proposed that growth and reproduction of animals is frequently limited by multiple nutrients simultaneously. To improve our understanding of the consequences of multiple nutrient limitations (i.e. co-limitation) for the performance of animals, we conducted standardized population growth experiments using an important aquatic consumer, the rotifer Brachionus calyciflorus.

2. We compared nutrient profiles (sterols, fatty acids and amino acids) of rotifers and their diets to reveal consumer-diet imbalances and thus potentially limiting nutrients. In concomitant growth experiments, we directly supplemented potentially limiting substances (sterols, fatty acids, amino acids) to a nutrient-deficient diet, the cyanobacterium Synechococcus elongatus, and recorded population growth rates.

3. The results from the supplementation experiments corroborated the nutrient limitations predicted by assessing consumer-diet imbalances, but provided more detailed information on co-limiting nutrients. While the fatty acid deficiency of the cyanobacterium appeared to be of minor importance, the addition of both cholesterol and certain amino acids (leucine and isoleucine) improved population growth rates of rotifers, indicating a simultaneous limitation by sterols and amino acids.

4. Our results add to growing evidence that consumers frequently face multiple nutrient limitations and suggest that the concept of co-limitation has to be considered in studies assessing nutrient-limited growth responses of consumers.
\end{abstract}

Key-words: consumer, consumer-diet imbalance, dietary mismatch, fatty acid, global change, lipid, nutrition, phytoplankton, tetrahymanol, zooplankton

\section{Introduction}

The concept of co-limitation, that is, a simultaneous limitation by multiple nutrients, has been frequently used to describe nutrient-limited growth responses of primary producers (Davidson \& Howarth 2007; Elser et al. 2007; Saito, Goepfert \& Ritt 2008; Harpole et al. 2011). As yet, however, it has been poorly applied to the nutrient-limited growth of animals (Martin-Creuzburg, Sperfeld \& Wacker 2009; Lukas, Sperfeld \& Wacker 2011; Sperfeld, MartinCreuzburg \& Wacker 2012), which besides elemental nutrients also rely on a number of essential biochemicals (e.g. Beenakkers, Van der Horst \& Van Marrewijk 1985). As

${ }^{*}$ Corresponding author. E-mail: Alexander.Wacker@uni-potsdam.de yet, experiments providing clear evidence for a co-limitation of an animal by two or more essential nutrients are scarce, in particular in regard to biochemical nutrients. Recently, using the freshwater herbivore Daphnia as a model organism, we have shown that the growth of this herbivore can be limited simultaneously by a low availability of dietary sterols and polyunsaturated fatty acids (PUFAs) (Martin-Creuzburg, Sperfeld \& Wacker 2009; Sperfeld, Martin-Creuzburg \& Wacker 2012). Both sterols and PUFAs are indispensable structural components of cell membranes (Hulbert \& Else 2005; Hulbert et al. 2005) and both serve as precursors for a number of bioactive molecules. Sterols, for instance, are precursors of steroid hormones, such as ecdysteroids, which in arthropods are involved in the process of moulting (Grieneisen 1994). Long-chain PUFAs, such as arachidonic acid (ARA) and 
eicosapentaenoic acid (EPA), are precursors of eicosanoids, which are thought to be relevant in invertebrate reproduction, the immune system and other physiological processes (Stanley-Samuelson 1994; Heckmann et al. 2008).

In aquatic ecosystems, a low availability of essential lipids and consequently a lipid limitation of zooplankton has been supposed to be associated with a high share of cyanobacteria within the phytoplankton (Müller-Navarra et al. 2000; Von Elert, Martin-Creuzburg \& Le Coz 2003), because cyanobacteria lack sterols and long-chain PUFAs (> C18) (Volkman 2003; Summons et al. 2006; MartinCreuzburg, Von Elert \& Hoffmann 2008). As cyanobacterial bloom formation, with all its negative effects on water quality, is presumably favoured by global warming (Jöhnk et al. 2008; Paerl \& Huisman 2008), a limitation of zooplankton consumers by essential lipids may be intensified in near future, in particular in eutrophic systems (Sperfeld \& Wacker 2009).

In contrast to fatty acids and sterols, the relevance of amino acids as potentially limiting nutrients has been mostly neglected in food quality studies. However, it has been shown that the availability of certain dietary amino acids correlates with the fecundity and hatching success of marine copepods (Guisande, Maneiro \& Riveiro 1999; Guisande, Riveiro \& Maneiro 2000; Helland et al. 2003) and a potential limitation of copepods by a low availability of the amino acid arginine has been suggested by Anderson, Boersma \& Raubenheimer (2004) based on comparisons between amino acid profiles of copepods and their food. Moreover, it has been suggested that specific dietary amino acids, in particular arginine and histidine, are involved in triggering the switch between reproductive modes in Daphnia, that is, they have been found to avert crowding-induced resting egg production and to enhance subitaneous reproduction (Koch et al. 2011). As amino acid profiles of natural food sources vary seasonally, (Cowie \& Hedges 1992; Petersson \& Floderus 2001; Kalachova et al. 2004), the performance of freshwater herbivores might be constrained not only by a low availability of essential dietary lipids, but also by the availability of certain amino acids, which potentially results in a simultaneous limitation by multiple nutrients.

Rotifers are important components of freshwater food webs (Walz 1995) that can dominate the zooplankton with densities often $>1000$ individuals per litre (Modenutti 1994; Dagne et al. 2008). By feeding on bacteria, algae, heterotrophic nanoflagellates and ciliates (Pourriot 1977; Sanders et al. 1989; Arndt 1993) and by serving as food, for example, for copepods and the larvae of insects and fishes, they are significantly involved in transferring energy and nutrients to higher trophic levels (Williamson 1983; Walsh 1995; Walz 1995). However, the efficiency with which rotifers use assimilated carbon for biomass production is potentially constrained by a low availability of essential nutrients, that is, elements (Rothhaupt 1995; Urabe, Clasen \& Sterner 1997; Jensen \& Verschoor 2004) or PUFAs
(Weithoff \& Wacker 2007; Wacker \& Weithoff 2009). Thus, given their high ecological relevance for food web processes rotifers are, besides Daphnia, promising model organisms to investigate multiple resource limitations and to deepen our knowledge on the concept of co-limitation.

Here, using the rotifer Brachionus calyciflorus as a model organism, we tested our hypothesis that also rotifers can be limited by multiple nutrients simultaneously. In feeding experiments, we compared nutrient profiles (sterols, fatty acids and amino acids) of rotifers and their diets to detect consumer-diet imbalances and thus potentially limiting nutrients. In addition, we conducted population growth experiments with rotifers feeding on a nutrient-deficient diet, which was supplemented with cholesterol, the most prominent sterol in animal tissues and/or EPA, either in the absence or in the presence of an additional source of amino acids. The significance of single dietary amino acids for the performance of rotifers was assessed by experimentally increasing the availability of several putatively essential dietary amino acids.

\section{Materials and methods}

\section{CULTIVATION OF FOOD ORGANISMS}

The coccoid cyanobacterium Synechococcus elongatus (SAG 89.79; culture collection of the University of Göttingen) and the flagellate Chlamydomonas reinhardtii (SAG 11-32b) were cultured semicontinuously in modified Woods Hole Culture Medium (WC) with vitamins (Guillard 1975) at $20{ }^{\circ} \mathrm{C}$ and an illumination of 30 and $120 \mu \mathrm{mol} \mathrm{m}^{-2} \mathrm{~s}^{-1}$ (16-h : 8-h light/dark cycle) in aerated 1-L vessels, respectively. The molar ratio of carbon to nitrogen to phosphorus $(\mathrm{C} / \mathrm{N}: \mathrm{P})$ of $S$. elongatus $(130: 16.5: 1)$ was close to the Redfield Ratio, indicating a high nitrogen and phosphorus content of the food suspensions. Thus, a limitation by nitrogen or phosphorus was highly improbable.

The carbon concentrations of the food stock suspensions were estimated from photometric light extinctions (at $800 \mathrm{~nm}$ ) using carbon-extinction equations determined prior to the experiment. Aliquots of algal or cyanobacterial stock suspensions were added to sterile WC medium, and the resulting suspensions of $2 \mathrm{mg} \mathrm{C} \mathrm{L}^{-1}$ were used as food/culture medium for rotifers. $S$. elongatus was used because it is a nontoxic, phosphorus-rich cyanobacterium that has been shown to be of poor food quality for Daphnia due to the lack of sterols and long-chain PUFAs (Fig. 1; Wacker \& Martin-Creuzburg 2007; Martin-Creuzburg, Sperfeld \& Wacker 2009).

\section{LIPOSOME PREPARATION}

The liposome stock solution was prepared from $3 \mathrm{mg}$ 1-palmitoyl2-oleoyl-phosphatidylglycerol (POPG) and $7 \mathrm{mg}$ 1-palmitoyl-2oleoyl-phosphatidylcholin (POPC; Lipoid, Ludwigshafen, Germany) dissolved in an aliquot of ethanol. Either $3.33 \mathrm{mg}$ of cholesterol or EPA (Sigma Aldrich, Deisenhofen, Germany) was added from a cholesterol or EPA solution in ethanol. Solutions were dried using a rotary evaporator and dissolved in $10 \mathrm{~mL}$ of buffer (15 mM [ $N$-Tris(hydroxymethyl)methyl-2-aminoethanesulfonic acid, TES], $150 \mathrm{~mm} \mathrm{NaCl}, \mathrm{pH} \mathrm{7.0)}$. Afterwards, the liposome suspension was sonicated in an ultrasonic bath for $30 \mathrm{~s}$ and excess free cholesterol or EPA was removed by washing the liposomes in fresh buffer using an ultra-speed centrifuge $(15.000 \mathrm{~g}, 360 \mathrm{~min}$, 


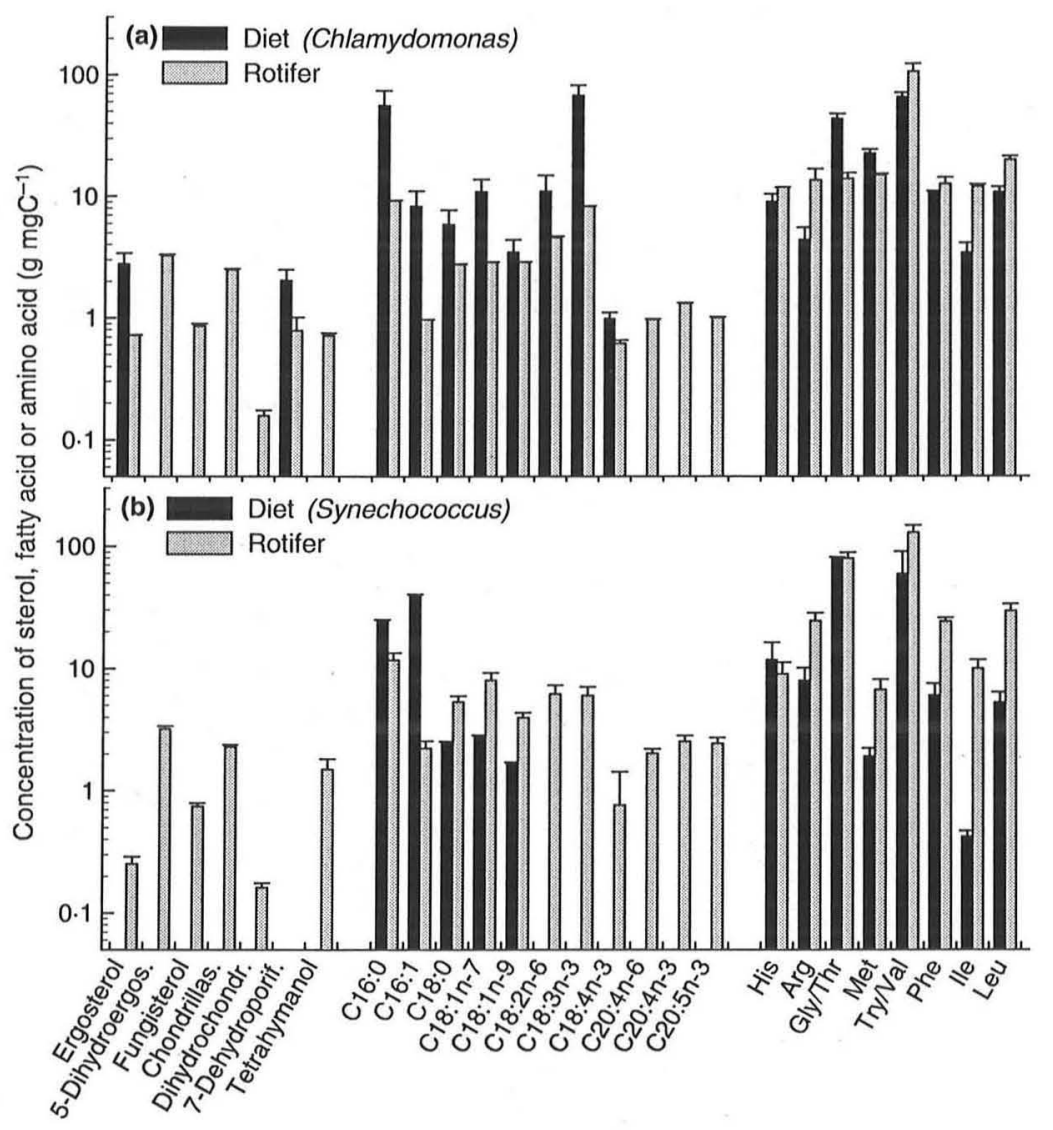

Fig. 1. Concentrations of sterols, tetrahymanol, fatty acids, and essential amino acids in (a) Chlamydomonas reinhardtii and Brachionus calyciflorus Pallas fed C. reinhardtii, and (b) Synechococcus elongatus and B. calycifforus fed S. elongatus (mean \pm standard deviation, $n=3$ ). Concentrations are given in log-scale.

$4{ }^{\circ} \mathrm{C}$ ). In addition, control liposomes without further ingredients were prepared. All liposome suspensions were stored at $-25^{\circ} \mathrm{C}$ until preparation of food treatments. The cholesterol and EPA content of liposomes was determined from subsamples; $1 \mu \mathrm{L}$ of the liposome suspensions contained either $0.52 \pm 0.02 \mu \mathrm{g}$ cholesterol or $0.17 \pm 0.01 \mu \mathrm{g} \mathrm{EPA} \mathrm{(mean} \pm \mathrm{SD}$ ), respectively. The mean diameter of liposomes was $2 \cdot 1-2 \cdot 2 \mu \mathrm{m}$ (determined by a particle counter; CASY, Schärfesystem, Germany)

\section{CULTIVATION OF ROTIFERS}

The rotifer strain (B. calyciflorus Pallas) that we used in the reported experiments was originally obtained from K.-O. Rothhaupt (University of Konstanz). Since then, this strain has been used as a 'rotifer model' in numerous studies (e.g. Rothhaupt 1988; Fussmann, Weithoff \& Yoshida 2005). Prior to the experiment, the rotifers were raised in glass beakers filled with $200 \mathrm{~mL}$ of sterile WC medium with saturating concentrations of C. reinhardtii $\left(>3 \mathrm{mg} \mathrm{C} \mathrm{L}^{-1}\right.$ ), to standardize experimental conditions and to start all experiments with animals in comparable nutritional state; no mixis was observed neither during preculturing nor during the experiments. Before diet changes, rotifers were repeatedly sieved using a $55-\mu \mathrm{m}$ mesh and rinsed with sterile culture medium to remove food organisms. To obtain sufficient biomass for chemical analyses, rotifers were reared in semicontinuous cultures in $700 \mathrm{~mL}$ of WC at a density of $c .10$ B. calyciflorus per millilitre and fed either with $C$. reinhardtii or S. elongatus. Fresh food suspensions of $C$. reinhardtii or $S$. elong- atus were provided daily so that the food concentration in cultures was c. $2 \mathrm{mg} \mathrm{C} \mathrm{L}^{-1}$.

\section{ROTIFER GROWTH EXPERIMENTS}

To standardize experimental conditions, growth experiments were conducted with newly hatched juveniles that hatched in $S$. elongatus without supplementations. Juveniles were collected and transferred randomly to the different treatments. Experiments lasted 6 days and were conducted at $20^{\circ} \mathrm{C}$ in microtitre wells filled with $10 \mathrm{~mL}$ food/culture suspension.

Prior to the co-limitation experiments, we conducted preliminary growth experiments using (i) WC medium without any algal diet, (ii) as before but with control liposomes, (iii) WC medium enriched with $25 \mathrm{mg} \mathrm{L}^{-1}$ dissolved bovine serum albumin (BSA; Sigma-Aldrich) to provide an unspecific source of amino acids, (iv) the latter combined with liposomes and (v) C. reinhardtii as a reference food with a carbon concentration of $2 \mathrm{mg} \mathrm{C} \mathrm{L}^{-1}$. Without any particulate food sources such as $C$. reinhardtii or $S$. elongatus, all rotifers died within 4 days irrespective of whether they were kept on pure WC medium or on pure WC medium supplemented with BSA and/or liposomes (Fig. S1, Supporting Information). Previous tests also revealed that different concentrations of control liposomes, $0.33,0.66,1.3 \mu \mathrm{L} \mathrm{mL}^{-1}$ food suspension containing $2 \mathrm{mg} \mathrm{C} \mathrm{L}^{-1} S$. elongatus, did not significantly affect growth rates of rotifers.

In the first co-limitation experiment, rotifers were fed $S$. elongatus $\left(2 \mathrm{mg} \mathrm{C} \mathrm{L}^{-1}\right)$ supplemented with cholesterol and/or 
EPA-containing liposomes either in the absence or in the presence of BSA, that is, an additional source of amino acids. C. reinhardtii was used as a reference food. Liposome supplemented diets were prepared by adding $0.167 \mu \mathrm{L}$ of liposome stock suspensions to $1 \mathrm{~mL}$ of food suspension resulting in $87 \mathrm{ng}$ cholesterol $\mathrm{mL}^{-1}$ and/ or $28 \mathrm{ng}$ EPA mL ${ }^{-1}$ food suspension. This equals $43 \mu \mathrm{g}$ cholesterol and $14 \mu \mathrm{g}$ EPA $\mathrm{mg}^{-1}$ cyanobacterial carbon, respectively. BSA supplemented diets were prepared by adding $25 \mu \mathrm{g}$ dissolved BSA to $1 \mathrm{~mL}$ of food suspension.

In the second co-limitation experiment, rotifers were fed either unsupplemented $S$. elongatus or $S$. elongatus supplemented with one of 10 putatively essential amino acids ( $25 \mu \mathrm{M}$ of food suspension). A previous test showed that amino acid concentrations of $25 \mu \mathrm{M}$ had no detrimental effect on rotifers but were in a sufficient range to induce growth enhancing effects. Amino acids are abbreviated as follows: histidine, His; arginine, Arg; threonine, Thr; methionine, Met; valine, Val; phenylalanine, Phe; isoleucine, Ile; leucine, Leu; lysine, Lys and tryptophane, Try. C. reinhardtii was again used as a reference food.

The initial density of rotifers in all growth experiments was one rotifer per millilitre culture medium. At 24-h intervals, the food suspensions were completely renewed and the rotifers were counted under a stereo microscope. To avoid density dependent processes, 10 rotifers were randomly chosen and pipetted into new food suspensions of $10 \mathrm{~mL}$. In case of negative population growth rates, all remaining individuals were transferred into new food suspensions. Densities of rotifers in all treatments fluctuated below those reported to cause consumer-dependent effects by direct and indirect consumer interference (Fussmann, Weithoff \& Yoshida 2005). After 2 days of acclimatization, the numbers of individuals were used to calculate intrinsic growth rates for daily intervals using the equation:

$r=\ln \left(N_{t}\right)-\ln \left(N_{t-1}\right)$

where $r$ is the intrinsic growth rate (per day), and $N_{t}$ and $N_{t}$ are the animal numbers on consecutive days. The population growth rate of each replicate and treatment was estimated by calculating the average value of the intrinsic growth rates of rotifers on consecutive days.

\section{ANALYTICAL PROCEDURES}

For analytical purposes, rotifers fed with C. reinhardtii were sieved using a $55-\mu \mathrm{m}$ mesh and repeatedly resuspended and rinsed with sterile culture medium to remove food organisms. Then rotifers were transferred to the target food suspensions $C$. reinhardtii and $S$. elongatus $\left(2 \mathrm{mg} \mathrm{C} \mathrm{L}^{-1}\right)$. Each rotifer culture was grown for 3 days with food suspensions renewed on the second day. The cultures were sampled 1 day after the last supply of food to minimize potential contamination of the rotifer sterol, fatty acid and amino acid profiles by undigested food particles from the digestive tract. Again the rotifers were sieved, repeatedly re-suspended and rinsed with sterile culture medium. Subsamples of rotifers were counted under a stereo microscope to determine rotifer densities, and aliquots containing 7500 individuals were filtered onto a 25 $\mathrm{mm}$ glass-fibre filter (Whatman, GF/C; Whatman International Ltd, Maidstone, UK). Likewise, aliquots of the $C$. reinhardtii and $S$. elongatus food suspensions, corresponding to 0.5 or $1.0 \mathrm{mg}$ particulate organic carbon (POC), were filtered onto Whatman GF/F filters $(25 \mathrm{~mm})$. Prior to extraction of sterols and fatty acids, $10 \mu \mathrm{g}$ of $5 \alpha$-cholestan or $20 \mu \mathrm{g}$ of tricosanoic acid methyl ester, respectively (Sigma-Aldrich), were added as internal standards. Filters were extracted with $7 \mathrm{~mL}$ dichloromethane/methanol (2: 1 v: v) and stored under nitrogen at $-25{ }^{\circ} \mathrm{C}$ until analysis. Fatty acids and sterols of diets and rotifers were analysed according to Wacker \& Weithoff (2009) and Martin-Creuzburg, Sperfeld \&
Wacker (2009), respectively, and normalized to the carbon content of the samples.

Prior to analysis of amino acids, the samples (stored under nitrogen at $-80^{\circ} \mathrm{C}$ until analysis) were hydrolysed with $6 \mathrm{M} \mathrm{HCl}$ at $110^{\circ} \mathrm{C}$ for $24 \mathrm{~h}$, neutralized with $6 \mathrm{M} \mathrm{NaOH}$ and analysed according to Gzik (1996). The hydrolysed and lyophilized samples were dissolved in $80 \%$ methanol and aliquots were derivatized

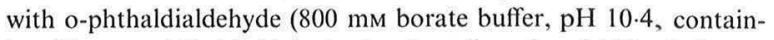
ing $50 \mathrm{mg}$ o-phthaldialdehyde, $1 \mathrm{~mL}$ methanol and $100 \mu \mathrm{L} \mathrm{3-mer-}$ captopropionic acid) to yield stable, fluorescent derivatives. After a reaction time of $2 \mathrm{~min}$, derivatives were separated by reversedphase high-performance liquid chromatography (Knauer $\mathrm{GmbH}$, Berlin, Germany) using an Aqua(B) column $(5 \mu \mathrm{m} \mathrm{C18} 125 \AA$ LC, $250 \times 4.6 \mathrm{~mm}$ id; Phenomenex Inc., Torrance, CA, USA), and a stepwise gradient of acetate buffer ( $\mathrm{pH}$ 7.0) with increasing concentration of methanol. Eluents were detected using a fluorescence detector (Model RF 551; Shimadzu, Kyoto, Japan). With this method, Try and Val as well as Gly and Thr, respectively, could not be separated. Amino acids were quantified using multipoint standard calibration curves previously determined for each amino acid and normalized to the carbon content of the sample.

For the determination of POC and nitrogen aliquots containing 1500 B. calyciflorus or $0.5 \mathrm{mg}$ POC of C. reinhardtii and S. elongatus were filtered onto precombusted glass-fibre filters $(25 \mathrm{~mm}$ diameter; Whatman GF/F) and after drying for at least 2 days at $50{ }^{\circ} \mathrm{C}$ analysed for carbon and nitrogen using an elemental analyser (EuroEA 3000; HEKAtech GmbH, Wegberg, Germany). For the determination of particulate organic phosphorus concentrations, aliquots of food suspensions were filtered onto polysulphone filters ( $25 \mathrm{~mm}, 0.45 \mu \mathrm{m}$; Pall Corporation, Ann Arbor, MI, USA) and analysed using the molybdate-ascorbic acid method (Murphy \& Riley 1962) after dissolving tissues with sulphuric acid and oxidative hydrolysation by $\mathrm{K}_{2} \mathrm{~S}_{2} \mathrm{O}_{8}$ at $120^{\circ} \mathrm{C}$ for $60 \mathrm{~min}$.

\section{DATA ANALYSES}

Differences in population growth rates were analysed by two-way ANOVA (co-limitation experiment 1) or one-way ANOva (co-limitation experiment 2) with subsequent post hoc comparisons (Tukey's HSD), when a factor or an interaction was significant $(P<0.05)$. Main factors were either BSA treatment and type of liposome treatment (co-limitation experiment 1) or amino acid treatment (co-limitation experiment 2). Normality was tested using the Shapiro-Wilk's test and homogeneity of variances using FlignerKilleen's test. Additionally, statistical models were tested for linearity and constant variances. According to Crawley (2002), data of co-limitation experiment 2 were boxcox transformed with lambda $=3$, that is, by using $\left((1+y)^{3}-1\right) / 3$ to meet assumptions. All calculations and statistical tests were conducted with the software package $R$, which is under general public licence ( $R$ Project for Statistical Computing, www.R-project.org).

\section{Results}

\section{CONSUMER-DIET IMBALANCE IN NUTRIENTS}

Considerable consumer-diet imbalances were detected for sterols, some n-3 PUFAs and in particular certain amino acids (Fig. 1), suggesting a dietary deficiency and thus a potential limitation of rotifer growth by these individual dietary compounds. The sterol composition of rotifers fed C. reinhardtii was characterized by ergosterol (IUPAC name; (22E)-ergosta-5,7,22-trien-3 $\beta$-ol), 5-dihydroergosterol [(22E)$5 \alpha$-ergosta-7,22-dien-3 $\beta$-ol], fungisterol ( $5 \alpha$-ergost-7-en-3 $\beta$-ol), 


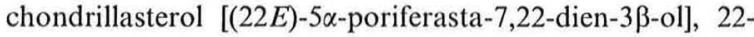
dihydrochondrillasterol ( $5 \alpha$-poriferast-7-en- $3 \beta$-ol), and 7-dehydroporiferasterol [(22E)-poriferasta-5,7,22-trien-3 $\beta$-ol]. Ergosterol and 7-dehydroporiferasterol were the principal sterols detected in C. reinhardtii and thus were presumably of dietary origin; the other sterols detected in the rotifers were absent from the diet. All these sterols, except 7-dehydroporiferasterol, were also detected in rotifers fed the sterol-free $S$. elongatus (Fig. 1), which suggest that these sterols were either synthesized de novo or originated from preexperimental feeding and were retained in the animals, (Fig. 2). This consumer-diet imbalance was evident for all sterols detected in the rotifers. In addition to sterols, the pentacyclic triterpenoid alcohol tetrahymanol was detected in the rotifers. Tetrahymanol was detected in somewhat higher concentrations in animals fed the sterol-free $S$. elongatus than in animals fed the sterol-containing $C$. reinhardtii.

The rotifers contained several long-chain PUFAs that were absent from the diets (Fig. 1). For instance, rotifers fed $C$. reinhardtii contained significant amounts of $\mathrm{C} 20$ PUFA that could not be detected in $C$. reinhardtii. After the diet switch to $S$. elongatus, a comparison of PUFA compositions revealed an additional consumer-diet imbal-

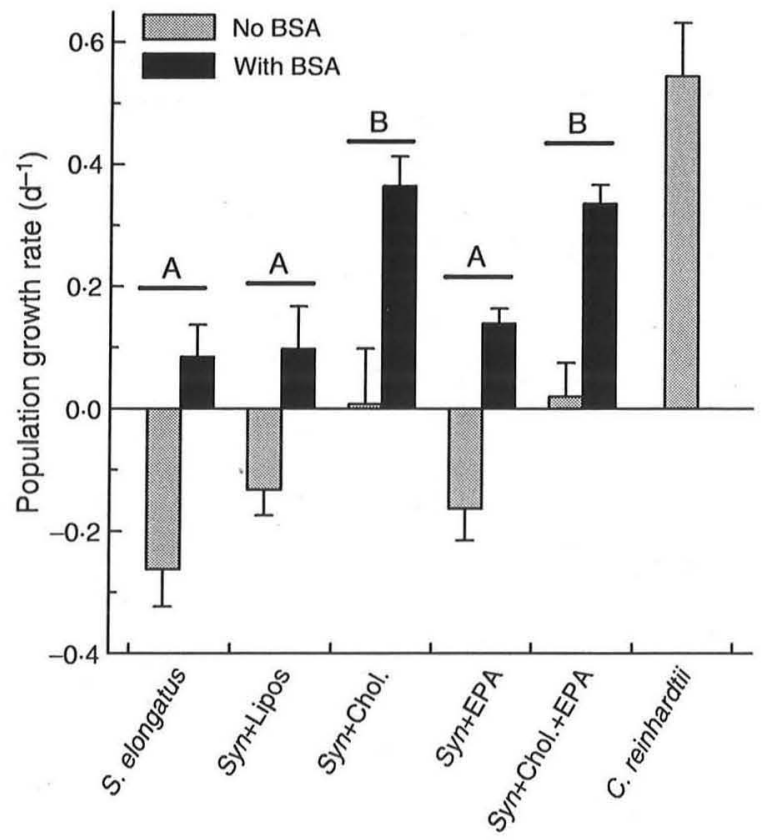

Fig. 2. Population growth rates (means \pm standard deviations, $n=4$ ) of Brachionus calyciflorus Pallas raised on different food regimes in a two-factorial design. One factorial treatment was the dietary presence of bovine serum albumin (BSA) in the food suspensions and the second factor was the treatment with different kinds of lipids supplemented via liposomes. Treatment with BSA has a significant effect on growth (ANOVA, $P<0.001$ ), regardless of any interaction with the liposome supplementations. Since there was no interaction between both factors, bars with different letters (A or B) display significant different levels of factor two, that is, differences between lipid supplementations $(P<0.001$; Tukey HSD following two-way ANOva). ance in $\mathrm{Cl} 8 \mathrm{PUFAs}$, in particular in $\alpha$-linolenic acid (ALA) and linoleic acid (LIN) (Fig. 1). Except for small differences in the concentrations of Arg and Ile, the amino acid compositions and concentrations did not differ between $C$. reinhardtii and rotifers fed $C$. reinhardtii. In contrast, a comparison of amino acid concentrations of $S$. elongatus and rotifers fed $S$. elongatus revealed a high consumer-diet imbalance in Ile, followed by a somewhat smaller imbalance in Leu, Met, Phe and Arg, suggesting a potential growth limitation by these dietary amino acids.

\section{CO-LIMITATION EXPERIMENT 1}

Population growth rates of rotifers significantly increased upon BSA supplementation by c. $0 \cdot 3$ day $^{-1}$ (differences between means; Fig. 2; Table 1), irrespective of simultaneous supplementation with control liposomes or EPAcontaining liposomes. Similarly, population growth rates significantly increased upon supplementation with cholesterol-containing liposomes by c. $0.25 \mathrm{day}^{-1}$, irrespective of simultaneous supplementation with BSA (Fig. 2; Table 1, Tukey's HSD, $P<0.001$ following ANOVA). Population growth rates significantly increased also by the simultaneous supplementation with cholesterol- and EPA-containing liposomes, but the obtained growth rates did not differ significantly from those obtained by cholesterol supplementation alone. The supplementation with control liposomes or EPA-containing liposomes did not significantly increase population growth rates (Tukey's HSD, $P>0 \cdot 19)$.

\section{CO-LIMITATION EXPERIMENT 2}

Population growth rates of rotifers fed S. elongatus increased significantly upon isoleucine and leucine supplementation (Fig. 3, Table 1, Tukey's HSD, $P<0.01$ and $P<0.05$, respectively). All other amino acids did not significantly improve population growth rates compared to animals fed unsupplemented $S$. elongatus (Fig. 3, Tukey's HSD, $P>0.9$ ). Growth rates increased upon isoleucine and leucine supplementation by 0.29 and 0.25 day $^{-1}$ compared with unsupplemented S. elongatus (Fig. 3, Table 1), that is, in a similar range as observed upon BSA supplementation in co-limitation experiment 1 (Fig. 2).

\section{Discussion}

Our study shows that population growth of the rotifer $B$. calyciflorus can be limited by more than just one nutrient simultaneously, that is, by sterols and certain amino acids, which adds to growing evidence that multiple resource limitation of consumers is a common phenomenon in aquatic ecosystems. We used a controlled laboratory system with unialgal diets to control, for example, prey selection and other confounding factors potentially associated with the diversity of natural algal diets. 
Table 1. Statistical analysis of the obtained population growth rates of Brachionus calyciflorus Pallas raised on different diets

\begin{tabular}{|c|c|c|c|c|}
\hline \multirow[b]{2}{*}{ Experiment } & \multirow[b]{2}{*}{ Factor } & \multicolumn{3}{|c|}{ Population growth rate (per day) } \\
\hline & & d.f. & $F$-value & $P$-value \\
\hline \multirow[t]{3}{*}{ Co-limitation experiment 1} & Protein treatment (BSA) & 1,30 & $312 \cdot 3$ & $<0.0001$ \\
\hline & Liposome treatments (Lipo) & 4,30 & $26 \cdot 59$ & $<0.0001$ \\
\hline & BSA $\times$ Lipo & 4,30 & 1.63 & $0 \cdot 19$ \\
\hline Co-limitation experiment 2 & Amino acid treatment (AA) & 10,33 & 6.73 & $<0.0001$ \\
\hline
\end{tabular}

In co-limitation experiment 1 , differences in population growth rates were analysed by two-way ANovA. The factor 'liposome treatment' includes different factor levels consisting of different kinds of lipids supplemented to the diet via liposomes. The factor "bovine serum albumin (BSA) treatment' represents the presence or absence of BSA in the food suspensions. In co-limitation experiment 2 , the factor 'amino acid treatment' represents the presence or absence of single amino acids in the food suspensions (one-way design).

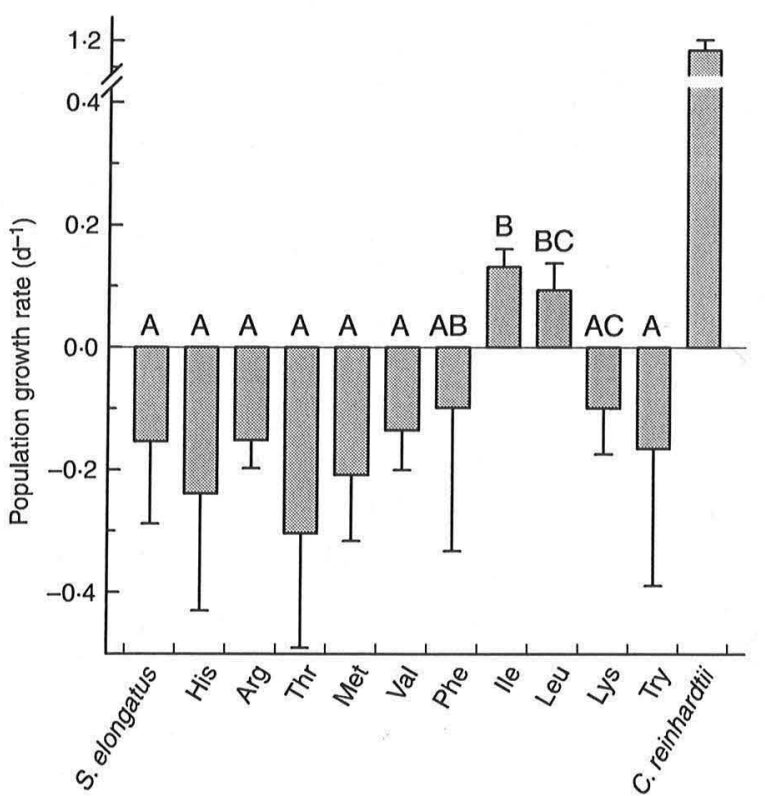

Fig. 3. Population growth rates (mean \pm standard deviation, $n=4$ ) of Brachionus calyciflorus Pallas raised on Synechococcus elongatus supplemented with one of 10 different amino acids. Bars with different letters display significant differences between amino acid supplementations $(P<0.05$; Tukey HSD following one-way ANOVA).

Population growth rates of $B$. calyciflorus increased significantly upon cholesterol supplementation, indicating that a dietary source of sterols is required to obtain high population growth rates. As yet, our knowledge on sterol requirements of rotifers was based merely on correlative evidence. Boechat \& Adrian (2006) found that egg production, but not population growth, of the rotifer Keratella quadrata was correlated with the dietary sterol content and concluded that a dietary source of sterols is required for rotifer reproduction. In the present study, we provide experimental evidence clearly showing that population growth rates of rotifers are constrained by a low availability of dietary sterols.

Teshima et al. (1981) reported that the rotifer Brachionus plicatilis is capable of synthesizing cholesterol, but not C28- and C29-sterols, from radio-labelled acetate, but at the same time argued that cholesterol and the C28- and C29- sterols detected were mostly of dietary origin. In our study, we did not find any cholesterol in B. calyciflorus but several C28- and C29-sterols, suggesting that cholesterol is not synthesized by $B$. calyciflorus even under sterol limitation. One would expect that the putative sterol synthesising pathway is highly conserved among rotifers and that, therefore, the two species B. plicatilis and B. calyciflorus should have similar capabilities. However, although we cannot definitely infer from our data whether B. calyciflorus is capable of synthesising sterols de novo, the growth enhancing effect of sterol supplementation suggests that the animals are either incapable of synthesising sterols $d e$ novo or that the rates of synthesis are too low to meet physiological demands. The finding that B. calyciflorus maintained relatively high body sterol levels even in the absence of dietary sterols, that is, when fed unsupplemented $S$. elongatus, implies that sterols obtained from dietary sources provided prior to the experiment (i.e. from $C$. reinhardtii) were retained and partially modified within their body. However, specific time course experiments will be required to accurately assess the capability of rotifers to retain and metabolize dietary sterols. Consequently, the animals might be highly affected by changes in the sterol availability, which depends on the phytoplankton composition and environmental conditions (Piepho, Martin-Creuzburg \& Wacker 2010).

In addition to sterols, the pentacyclic triterpenoid alcohol tetrahymanol was detected in the rotifers, although it was absent from the diet, suggesting that rotifers are capable of synthesising tetrahymanol de novo. In ciliates, tetrahymanol is produced as sterol surrogate in the absence of dietary sterols and has been shown to be functionally equivalent to sterols as a structural component of cell membranes (Ourisson, Rohmer \& Poralla 1987; Harvey \& McManus 1991; Martin-Creuzburg, Bec \& Von Elert 2006). To our knowledge, this is the first time that tetrahymanol has been detected in a rotifer. The tetrahymanol content of rotifers was not significantly affected by the availability of dietary sterols, suggesting that tetrahymanol cannot serve as sterol surrogate in rotifers, but this should be subjected to further investigations. 
Supplementation with EPA did not affect population growth rates of B. calyciflorus (Fig. 2), suggesting that a dietary source of EPA is not required. However, this is in contrast to previous studies reporting that egg production of the rotifer Keratella quadrata fed ciliates was correlated with the availability of dietary PUFAs, in particular EPA (Boechat \& Adrian 2006). Recently it has been shown that environmentally induced changes in dietary PUFA availabilities (Spijkerman \& Wacker 2011) strongly affect the growth of rotifer species from acidic mining lakes (Weithoff \& Wacker 2007) and their competitive abilities (Hartwich, Wacker \& Weithoff 2010). These contradictory findings suggest that different dietary constraints arise from feeding on cyanobacteria, as in the present study, and eukaryotic food sources, as in the cited studies.

The ecological relevance of a dietary deficiency in essential amino acids is potentially high, as suggested by large consumer-diet imbalances in certain amino acids found in several aquatic and terrestrial consumers (Anderson, Boersma \& Raubenheimer 2004). In general, the amino acid content of invertebrate (Øie \& Olsen 1997; Tidwell et al. 1998; Helland et al. 2003) and vertebrate (Alam et al. 2002) consumers tends to be regulated homoeostatically, that is, despite considerable changes in dietary amino acid availabilities, changes in the amino acid content of consumers are rather low. The tendency of consumers to maintain certain amino acid concentrations within their body may increase the risk of a limitation by single amino acids, in particular by those amino acids which are temporarily underrepresented in the diet (Kalachova et al. 2004). This assumption is supported by the observation that the hatching success of copepods is improved when differences in the amino acid composition between females and their diet are less pronounced (Guisande, Maneiro \& Riveiro 1999; Guisande, Riveiro \& Maneiro 2000; Helland et al. 2003). In the theoretical approach of Anderson, Boersma \& Raubenheimer (2004), a comparison of amino acid profiles of copepods and their phytoplankton prey revealed a significant consumer - diet imbalance for arginine and threonine, suggesting that the performance of copepods is potentially constrained by the dietary availability of these amino acids. Following the same approach, our data revealed considerable differences in the concentrations of certain amino acids, in particular leucine and isoleucine, between $S$. elongatus and rotifers fed $S$. elongatus, suggesting a potential growth limitation of rotifers by leucine and isoleucine (Fig. 1). This was corroborated by the amino acid supplementation experiment, clearly showing that the population growth of $B$. calyciflorus on S. elongatus was in fact constrained by the availability of leucine and isoleucine.

The hypothesis that isoleucine is of general importance for invertebrate growth and reproduction is supported by the finding that a lack of isoleucine in the diet of the Mediterranean fruit fly, Ceratitis capitata, resulted in lowest larval growth compared to the absence of other potentially essential amino acids, except for threonine and tryptophan where no larvae survived (Chang 2004). The beneficial effect of leucine on population growth of $B$. calyciflorus is in accordance with a previous study showing that egg production of the rotifer Keratella quadrata was correlated, among other biochemical nutrients (fatty acids and sterols), with the leucine content of its protist food source (Boechat \& Adrian 2006).

The availability of amino acids might correlate well with that of nitrogen, and nitrogen was suggested as surrogate measurement for amino acids (Jensen et al. 2006; Hessen et al. 2007). Therefore, one might speculate that the observed positive effects of BSA or amino acid supplementation are in fact due to the increased availability of nitrogen rather than to the addition of essential amino acids. However, in our direct supplementation approach we did not find evidence for a potential limitation of the rotifers by particularly nitrogen-rich amino acids, such as arginine or histidine. Only the addition of leucine and isoleucine (with low nitrogen contents) improved population growth rates of B. calyciflorus (Fig. 3), clearly indicating that the rotifers were limited by the low dietary availability of these essential biochemical molecules and not by the availability of dietary nitrogen. In general, the dietary supply of elemental nutrients was high in the present study and thus a limitation of $B$. calyciflorus by phosphorus or nitrogen appears unlikely. Moreover, the supplementation of S. elongatus with control liposomes, which provide an extra source of carbon and phosphorus (Martin-Creuzburg, Von Elert \& Hoffmann 2008), did not improve population growth rates, showing that phosphorus supply and food concentrations in general were sufficient. Hence, studies investigating nutritional requirements of rotifers should also consider essential biochemicals as potentially limiting nutrients and should not merely focus on insufficient dietary supplies of minerals such as phosphorus and nitrogen as has been done in the past (Rothhaupt 1995; Strojsova et al. 2010).

We show here, for the first time, that the rotifer $B$. calyciflorus relies on a dietary source of sterols and that a dietary sterol-deficiency results in reduced population growth rates, as indicated by a growth enhancing effect of sterol supplementation. Similarly, we show that population growth rates of $B$. calyciflorus, on the same nutrient-deficient diet, can be improved by amino acid (leucine, isoleucine) supplementation, indicating a simultaneous limitation by sterols and amino acids. The substantial increase in growth rates as a response to sterol and amino acid supplementation demonstrates that a dietary deficiency and not the production of toxins or growth inhibitors are responsible for the low food quality of S. elongatus. Rotifers significantly feed on cyanobacteria, even if these are toxic (Snell 1980; Walz 1995; Soares, Lurling \& Huszar 2010). Hence, our data suggest that the growth of rotifers is potentially constrained simultaneously by a deficiency in dietary sterols and certain amino acids. This might be particularly relevant when the phytoplankton is dominated by cyanobacteria. Although the addition 
of these biochemical compounds considerably improved the food quality of $S$. elongatus for B. calyciflorus, population growth rates obtained on the supplemented diets were significantly lower than those obtained on a sole diet of Chlamydomonas (Figs 2 and 3), suggesting that other food quality constraints become important once the sterol and amino acid requirements are met.

Although our approach is straightforward in identifying limiting dietary compounds it does not consider the possibility of using complementary food sources to obtain nutrient balanced diets or compensatory feeding, that is, the increased uptake of certain food sources to satisfy the requirements for single dietary nutrients (Raubenheimer \& Jones 2006). However, during extensive cyanobacteria blooms complementary and compensatory feeding might be of minor importance because rotifers potentially do not have sufficient alternative food sources and may depend on potential improvement of the sterol and amino acid availability through seasonal changes of the phytoplankton community.

We show here, for the first time, that an aquatic invertebrate is simultaneously constrained (co-limited) by a deficiency in dietary sterols and certain amino acids. Previous work using the same food organism reported a similar scenario for a completely distinct phylogenetic group of aquatic invertebrates (Crustaceae, Daphnia), which however was co-limited by sterols and fatty acids instead of amino acids. This suggests that (i) co-limitation among consumers is more widespread as expected, and that (ii) biochemical nutrient requirements differ among phylogenetic groups. A limitation of rotifers by these essential biochemical nutrients may also affect the functioning of freshwater food webs, as rotifers are significantly involved in the carbon transfer to higher trophic levels. Rotifers were successfully used to study mechanistic resource competition theory (Rothhaupt 1988) and for aquaculture purposes (Hagiwara et al. 1993; Makridis \& Olsen 1999; Aragao et al. 2004; Srivastava et al. 2006). The present study shows that they may also be of high value to study and characterize resource co-limitation scenarios.

\section{Acknowledgements}

We thank S. Donath, S. Heim, G. Gehrmann and P. Merkel for their technical assistance and two reviewers for their valuable comments. This study was supported by the German Research Foundation (DFG, WA2445).

\section{References}

Alam, M.S., Teshima, S., Yaniharto, D., Koshio, S. \& Ishikawa, M. (2002) Influence of different dietary amino acid patterns on growth and body composition of juvenile Japanese flounder, Paralichthys olivaceus. Aquaculture, 210, 359-369.

Anderson, T.R., Boersma, M. \& Raubenheimer, D. (2004) Stoichiometry: linking elements to biochemicals. Ecology, 85, 1193-1202.

Aragao, C., Conceicao, L.E.C., Dinis, M.T. \& Fyhn, H.J. (2004) Amino acid pools of rotifers and Artemia under different conditions: nutritional implications for fish larvae. Aquaculture, 234, 429-445

Arndt, H. (1993) Rotifers as predators on components of the microbial web (bacteria, heterotrophic flagellates, ciliates) - a review. IIydrobiologia, $255,231-246$.
Beenakkers, A.M.Th., Van der Horst, D.J. \& Van Marrewijk, W.J.A. (1985) Insect lipids and lipoproteins, and their physiological processes. Progress in Lipid Research, 24, 19-67.

Boechat, I.G. \& Adrian, R. (2006) Evidence for biochemical limitation of population growth and reproduction of the rotifer Keratella quadrata fed with freshwater protists. Journal of Plankıon Research, 28, 1027-1038.

Chang, C.L. (2004) Effect of amino acids on larvae and adults of Ceratitis capitata (Diptera: Tephritidae). Annals of the Entomological Society of America, 97, 529-535.

Cowie, G.L. \& Hedges, J.I. (1992) Sources and reactivities of amino acids in a coastal marine environment. Limnology \& Oceanography, 37, 703-724.

Crawley, M.J. (2002) Statistical Computing: An Introduction to Data Analysis Using S-Plus. John Wiley \& Sons, Chichester.

Dagne, A., Herzig, A., Jersabek, C.D. \& Tadesse, Z. (2008) A bundance, species composition and spatial distribution of planktonic rotifers and crustaceans in Lake Ziway (Rift Valley, Ethiopia). International Review of Hydrobiology, 93, 210-226.

Davidson, E.A. \& Howarth, R.W. (2007) Environmental science: Nutrients in synergy. Nature, 449, 1000-1001.

Elser, J.J., Bracken, M.E.S., Cleland, E.E., Gruner, D.S., Harpole, W.S., Hillebrand, H., Ngai, J.T., Seabloom, E.W., Shurin, J.B. \& Smith, J.E. (2007) Global analysis of nitrogen and phosphorus limitation of primary producers in freshwater, marine and terrestrial ecosystems. Ecology Letters, 10, 1135-1142.

Fussmann, G.F., Weithoff, G. \& Yoshida, T. (2005) A direct, experimental test of resource vs. consumer dependence. Ecology, 86, 2924-2930.

Grieneisen, M.L. (1994) Recent advances in our knowledge of ecdysteroid biosynthesis in insects and crustaceans. Insect Biochemistry And Molecular Biology, 24, 115-132

Guillard, R.R.L. (1975) Cultures of phytoplankton for feeding of marine invertebrates. Culture of Marine Invertebrate Animals (eds W.L. Smith \& M.H. Chanley), pp. 29-60. Plenum, New York.

Guisande, C., Maneiro, I. \& Riveiro, I. (1999) Homeostasis in the essentia amino acid composition of the marine copepod Euterpina acutifrons. Limnology and Oceamography, 44, 691-696.

Guisande, C., Riveiro, I. \& Maneiro, I. (2000) Comparisons among the amino acid composition of females, eggs and food to determine the relative importance of food quantity and food quality to copepod reproduction. Marine Ecology-Progress Series, 202, 135-142.

Gzik, A. (1996) Accumulation of proline and pattern of alpha-amino acids in sugar beet plants in response to osmotic, water and salt stress. Environmental and Experimental Botany, 36, 29-38.

Hagiwara, A., Hamada, K., Nishi, A., Imaizumi, K. \& Hirayama, K (1993) Dietary value of neonates from rotifer Brachionus plicatilis resting eggs for red-sea bream larvae. Nippon Suisan Gakkaishi, 59, 99-104.

Harpole, W.S., Ngai, J.T., Cleland, E.E., Seabloom, E.W., Borer, E.T. Bracken, M.E.S., Elser, J.J., Gruner, D.S., Hillebrand, H., Shurin, J.B. \& Smith, J.E. (2011) Nutrient co-limitation of primary producer communities. Ecology Letters, 14, 852-862.

Hartwich, M., Wacker, A. \& Weithoff, G. (2010) Changes in the competitive abilities of two rotifers feeding on mixotrophic flagellates. Journal of Plankton Research, 32, 1727-1731.

Harvey, H.R. \& McManus, G.B. (1991) Marine ciliates as a widespread source of tetrahymanol and hopan-3-beta-ol in sediments. Geochimica et Cosmochimica Acta, 55, 3387-3390.

Heckmann, L.H., Sibly, R., Timmermans, M. \& Callaghan, A. (2008) Outlining eicosanoid biosynthesis in the crustacean Daphnia. Frontiers in Zoology, 5, 11.

Helland, S., Nejstgaard, J., Humlen, R., Fyhn, H.J. \& Båmstedt, U. (2003) Effects of season and maternal food on Calamus finmarchicus reproduction, with emphasis on free amino acids. Marine Biology, 142, 1141-1151.

Hessen, D.O., Jensen, T.C., Kyle, M. \& Elser, J.J. (2007) RNA responses to $\mathrm{N}$ - and P-limitation; reciprocal regulation of stoichiometry and growth rate in Brachionus. Functional Ecology, 21, 956-962.

Hulbert, A.J. \& Else, P.L. (2005) Membranes and the setting of energy demand. Journal of Experimental Biology, 208, 1593-1599.

Hulbert, A.J., Turner, N., Storlien, L.H. \& Else, P.L. (2005) Dietary fats and membrane function: implications for metabolism and disease. Biological Reviews, 80, 155-169.

Jensen, T.C. \& Verschoor, A.M. (2004) Effects of food quality on life history of the rotifer Brachionus calyciflorus Pallas. Freshwater Biology, 49, $1138-1151$

Jensen, T.C., Anderson, T.R., Daufresne, M. \& Hessen, D.O. (2006) Does excess carbon affect respiration of the rotifer Brachionus calyciflorus Pallas? Freshwater Biology, 51, 2320-2333. 
Jöhnk, K.D., Huisman, J., Sharples, J., Sommeijer, B., Visser, P.M. \& Stroom, J.M. (2008) Summer heatwaves promote blooms of harmful cyanobacteria. Global Change Biology, 14, 495-512.

Kalachova, G.S., Kolmakova, A.A., Gladyshev, M.I., Kravchuk, E.S. \& Ivanova, E.A. (2004) Seasonal dynamics of amino acids in two small Siberian reservoirs dominated by prokaryotic and eukaryotic phytoplankton. Aquatic Ecology, 38, 3-15.

Koch, U., Martin-Creuzburg, D., Grossart, H.P. \& Straile, D. (2011) Single dietary amino acids control resting egg production and affect population growth of a key freshwater herbivore. Oecologia, 167, 981-989.

Lukas, M., Sperfeld, E. \& Wacker, A. (2011) Growth Rate Hypothesis does not apply across colimiting conditions: cholesterol limitation affects phosphorus homoeostasis of an aquatic herbivore. Functional Ecology, 25, 1206-1214.

Makridis, P. \& Olsen, Y. (1999) Protein depletion of the rotifer Brachionus plicatilis during starvation. Aquaculture, 174, 343-353.

Martin-Creuzburg, D., Bec, A. \& Von Elert, E. (2006) Supplementation with sterols improves food quality of a ciliate for Daphnia magna. Protist, 157, 477-486.

Martin-Creuzburg, D., Sperfeld, E. \& Wacker, A. (2009) Colimitation of a freshwater herbivore by sterols and polyunsaturated fatty acids. Proceedings of the Royal Society B: Biological Sciences, 276, 1805-1814.

Martin-Creuzburg, D., Von Elert, E. \& Hoffmann, K.H. (2008) Nutritional constraints at the cyanobacteria-Daphnia magna interface: The role of sterols. Limnology and Oceanography, 53, 456-468.

Modenutti, B.E. (1994) Spring summer succession of planktonic rotifers in a south-Andes lake. Internationale Revue der Gesamten Hydrobiologie, 79, 373-383.

Müller-Navarra, D.C., Brett, M.T., Liston, A.M. \& Goldman, C.R. (2000) A highly unsaturated fatty acid predicts carbon transfer between primary producers and consumers. Nature, 403, 74-77.

Murphy, J. \& Riley, J.P. (1962) A modified single solution method for determination of phosphate in natural waters. Analytica Chimica Acta, 26, 31-36.

Oie, G. \& Olsen, Y. (1997) Protein and lipid content of the rotifer Brachionus plicatilis during variable growth and feeding condition. Hydrobiologia, 358, 251-258

Ourisson, G., Rohmer, M. \& Poralla, K. (1987) Prokaryotic hopanoids and other polyterpenoid sterol surrogates. Annual Review of Microbiology, 41, 301-333.

Paerl, H.W. \& Huisman, J. (2008) Climate - blooms like it hot. Science, 320, 57-58.

Petersson, M. \& Floderus, S. (2001) Use of amino acid composition to investigate settling and resuspension of a spring bloom in the southern Skagerrak. Limnology \& Oceanography, 46, 1111-1120.

Piepho, M., Martin-Creuzburg, D. \& Wacker, A. (2010) Simultaneou effects of light intensity and phosphorus supply on the sterol content of phytoplankton. PLoS One, 5, el5828.

Pourriot, R. (1977) Food and feeding habits of Rotifera. Archiv für Hydrobiologie Beihefte Ergebnisse der Limnologie, 8, 243-260.

Raubenheimer, D. \& Jones, S.A. (2006) Nutritional imbalance in an extreme generalist omnivore: tolerance and recovery through complementary food selection. Animal Behaviour, 71, 1253-1262.

Rothhaupt, K.O. (1988) Mechanistic resource competition theory applied to laboratory experiments with zooplankton. Nature, 333, 660-662.

Rothhaupt, K.O. (1995) Algal nutrient limitation affects rotifer growth rate but not ingestion rate. Limnology \& Oceanography, 40, 1201-1208.

Saito, M.A., Goepfert, T.J. \& Ritt, J.T. (2008) Some thoughts on the concept of colimitation: Three definitions and the importance of bioavailability. Limnology and Oceanography, 53, 276-290.

Sanders, R.W., Porter, K.G., Bennett, S.J. \& Debiase, A.E. (1989) Seasonal patterns of bacterivory by flagellates, ciliates, rotifers, and cladocerans in a fresh water planktonic community. Limnology and Oceanography, 34. 673-687.

Snell, T.W. (1980) Blue green algae and selection in rotifer populations Oecologia, 46, 343-346.

Soares, M.C.S., Lurling, M. \& Huszar, V.L.M. (2010) Responses of the rotifer Brachionus calyciflorus to two tropical toxic cyanobacteria (Cylindrospermopsis raciborskii and Microcystis aeruginosa) in pure and mixed diets with green algae. Journal of Plankton Research, 32 999-1008.

Sperfeld, E., Martin-Creuzburg, D. \& Wacker, A. (2012) Multiple resource limitation theory applied to herbivorous consumers: Liebig's minimum rule versus interactive co-limitation. Ecology Letters, 15, 142-150.
Sperfeld, E. \& Wacker, A. (2009) Effects of temperature and dietary sterol availability on growth and cholesterol allocation of the aquatic keystone species Daphnia. Journal of Experimental Biology, 212, 3051-3059.

Spijkerman, E. \& Wacker, A. (2011) Interactions between P-limitation and different $\mathrm{C}$ conditions on the fatty acid composition of an extremophile microalga. Extremophiles, 15, 597-609.

Srivastava, A., Hamre, K., Stoss, J., Chakrabarti, R. \& Tonheim, S.K. (2006) Protein content and amino acid composition of the live feed rotifer (Brachionus plicatilis): With emphasis on the water soluble fraction. Aquaculture, 254, 534-543.

Stanley-Samuelson, D.W. (1994) The biological significance of prostaglandins and related eisosanoids in invertebrates. American Zoologist, 34, 589-598.

Strojsova, M., Suga, K., Hagiwara, A. \& Vrba, J. (2010) Effect of food quantity and quality on population growth rate and digestive activity in the euryhaline rotifer Brachionus plicatilis Müller. International Review of Hydrobiology, 94, 706-719.

Summons, R.E., Bradley, A.S., Jahnke, L.L. \& Waldbauer, J.R. (2006) Steroids, triterpenoids and molecular oxygen. Philosophical Transactions of the Royal Society B-Biological Sciences, 361, 951-968.

Teshima, S.I., Kanazawa, A., Kamezaki, N. \& Hirata, H. (1981) Fatty acids and sterol components of the rotifers cultured by a feedback system. Bulletin of the Japanese Society of Scientific Fisheries, 47, 515521

Tidwell, J.H., Webster, C.D., Coyle, S.D., Daniels, W.H. \& D’Abramo, L. R. (1998) Fatty acid and amino acid composition of eggs, muscle and midgut glands of freshwater prawns, Macrobrachium rosenbergii (de Man), raised in fertilized ponds, unfertilized ponds or fed prepared diets. Aquaculture Research, 29, 37-45.

Urabe, J., Clasen, J. \& Sterner, R.W. (1997) Phosphorus limitation of Daphnia growth: is it real? Limnology \& Oceanography, 42, 1436-1443.

Volkman, J.K. (2003) Sterols in microorganisms. Applied Microbiology and Biotechnology, 60, 495-506.

Von Elert, E., Martin-Creuzburg, D. \& Le Coz, J.R. (2003) Absence of sterols constrains carbon transfer between cyanobacteria and a freshwater herbivore (Daphnia galeata). Proceedings of the Royal Society of London Series B-Biological Sciences, 270, 1209-1214.

Wacker, A. \& Martin-Creuzburg, D. (2007) Allocation of essential lipids in Daphnia magna during exposure to poor food quality. Functional Ecology, 21, 738-747.

Wacker, A. \& Weithoff, G. (2009) Carbon assimilation mode in mixotroph and the fatty acid composition of their rotifer consumers. Freshwater Biology, 54, 2189-2199.

Walsh, E.J. (1995) Habitat-specific predation susceptibilities of a littoral rotifer to two invertebrate predators. Hydrobiologia, 313, 205-211.

Walz, N. (1995) Rotifer populations in plankton communities - energetics and life-history strategies. Experientia, 51, 437-453.

Weithoff, G. \& Wacker, A. (2007) The mode of nutrition of mixotrophic flagellates determines the food quality for their consumers. Functional Ecology, 21, 1092-1098.

Williamson, C.E. (1983) Invertebrate predation on planktonic rotifers. Hydrobiologia, 104, 385-396. 